\title{
Metodologias para o ensino de zoologia: uma análise bibliográfica reflexiva
}

A zoologia é a área da biologia que dedica seus estudos sobre os animais. Os estudantes aprendem sobre os animais durante todo ciclo da educação básica. Observa-se a importância de estudar sobre os animais devido a sua interação com a humanidade, sua importância ecológica, econômica e de saúde pública. Apesa de sua importância, nota-se que o ensino da zoologia apresenta diversos problemas, principalmente no que diz respeito ao processo de ensino e aprendizagem que pouco envolve atividade lúdicas, e baseia-se quase sempre em atividades conceituais. Desse modo, o objetivo de nossa pesquisa é realizar análise de literaturas publicadas sobre as metodologias para o ensino de zoologia na educação básica como meio de facilitar o processo de ensino. Como metodologia para a pesquisa, utilizou-se o site Google Scholar, analisando artigos publicados em menos de dez anos, por meio das seguintes palavras chaves: ensino; material didático; metodologia; zoologia. Nos artigos analisados, foi possível observar a utilização de modelos didáticos tridimensionais que simulam a estrutura corporal de animais, recurso multimídia, computadores com acesso à internet, fotografias de animais e/ou suas estruturas anatômicas, jogos didáticos e mapas conceituais. Diversos autores concordam que a adoção de recursos didáticos como estes facilitam o processo de aprendizagem além de tornar este mais interessante e atraente para o estudante. Assim, concluímos que a utilização de materiais didáticos que tornam mais palpáveis os conceitos apresentados em sala de aula, pode trazer mais qualidade para o processo de ensino nas escolas, além disso, é necessário que os educadores estejam sempre atualizando e capacitando-se para trazer inovações educacionais.

Palavras-chave: Ensino; Material didático; Metodologia; Zoologia.

\section{Methodologies for teaching zoology: a reflective bibliographic analysis}

\begin{abstract}
Zoology is the area of biology that dedicates its studies on animals. Students learn about animals throughout the basic education cycle. It is observed the importance of studying animals due to their interaction with humanity, their ecological, economic and public health importance. Despite its importance, it is noted that the teaching of zoology presents several problems, mainly with regard to the teaching and learning process, which involves little playful activity, and is almost always based on conceptual activities. Thus, the objective of our research is to carry out an analysis of published literature on the methodologies for teaching zoology in basic education as a means of facilitating the teaching process. As a research methodology, the Google Scholar website was used, analyzing articles published in less than ten years, using the following keywords: teaching; courseware; methodology; zoology. In the analyzed articles, it was possible to observe the use of threedimensional didactic models that simulate the body structure of animals, multimedia resources, computers with internet access, photographs of animals and/or their anatomical structures, didactic games and concept maps. Several authors agree that the adoption of teaching resources such as these facilitates the learning process, in addition to making it more interesting and attractive to the student. Thus, we conclude that the use of didactic materials that make the concepts presented in the classroom more palpable, can bring more quality to the teaching process in schools, in addition, it is necessary that educators are always updating and training themselves to bring educational innovations.
\end{abstract}

Keywords: Courseware; Methodology; Teaching; Zoology.

Topic: Práticas, Didática e Metodologias do Ensino

Reviewed anonymously in the process of blind peer.

Sulamita de Carvalho Lima

Universidade Federal do Rio de Janeiro, Brasil

http://lattes.cnpq.br/2520063099103178

http://orcid.org/0000-0002-3802-6076

sula.clima@ig.com.br

Jonatha Anderson Fraga Egidio

Universidade Estadual do Norte Fluminense Darcy Ribeiro, Brasil http://lattes.cnpq.br/9424766036531541

http://orcid.org/0000-0003-2212-877X

jonathaafegidio@gmail.com

Barbara Proença do Nascimento (iD

Universidade Federal do Rio de Janeiro, Brasil

http://lattes.cnpq.br/0737434153883680

http://orcid.org/0000-0001-8081-0676

bproenca28@gmail.com

d

DOI: 10.6008/CBPC2318-3047.2021.002.0005
Received: 19/04/2021

Approved: 20/07/2021
Referencing this:

LIMA, S. C.; EGIDIO, J. A. F.; NASCIMENTO, B. P.. Metodologias para o ensino de zoologia: uma análise bibliográfica reflexiva. Educationis, v.9, n.2, p.43-50, 2021. DOI: http://doi.org/10.6008/CBPC23183047.2021.002.0005 


\section{INTRODUÇÃO}

A zoologia é a ciência que estuda os animais, tanto as espécies vivas quanto as extintas. Esse tema é abordado, ao longo da vida acadêmica do aluno, durante a educação básica, nos ensinos fundamental e médio. No início do aprendizado, durante o ensino fundamental, o estudo dos animais é apresentado na área de Ciências da Natureza e as suas características são trabalhadas a partir das impressões que os alunos têm desses organismos. Esse estudo é ministrado dentro da temática 'Vida e evolução', uma das três unidades temáticas da área de Ciências da Natureza e que se repete ao longo de todo o Ensino Fundamental.

Nessa temática são estudadas diversas questões relacionadas aos animais, como os elementos essenciais à sua manutenção, suas interações com o meio ambiente e demais seres vivos e, também, à compreensão dos processos evolutivos que geram a diversidade de formas de vida no planeta. São abordados, ainda, aspectos que mostram a importância da preservação da biodiversidade e como ela se distribui nos principais ecossistemas brasileiros. No Ensino médio, a etapa final da Educação Básica, o estudo dos animais é abordado dentro da área de 'Ciências da natureza e suas tecnologias' que têm como objetivo o aprofundamento, ampliação e consolidação dos assuntos estudados durante a temática 'Vida e evolução' vista no ensino fundamental.

Segundo Bessa et al. (2018) essa base curricular traz algumas vantagens, porém diversos problemas como, por exemplo, ir na direção oposta às principais propostas de melhorias na educação, como a flexibilidade no conteúdo, velocidade e modo do aprendizado. Os autores também destacam que aspectos importantes da zoologia não serão abordados, pois essa disciplina será trabalhada apenas no primeiro ciclo do ensino fundamental, no 20 e 3 o anos, e depois será abordada novamente apenas no ensino médio. Ou seja, por oito anos, durante boa parte de sua formação e construção da personalidade, os alunos não terão contato com a zoologia, e, quando tiverem, essa disciplina será dada de uma forma pouco abrangente, visto à grande biodiversidade que nosso país apresenta.

Outra problemática com relação ao ensino da zoologia, além do conteúdo pedagógico, são as ferramentas didáticas utilizadas, como, por exemplo, o uso exclusivo do livro didático pelos professores, utilização apenas da exposição oral como recurso educativo e falta de recursos didáticos alternativos. Além disso, muitos professores têm tempo reduzido para planejar e realizar suas atividades acadêmicas em sala de aula, laboratório e espaços não formais (SANTOS et al., 2017; ARAÚJO, 2011). É importante que no ensino da zoologia, os professores saiam um pouco da sala de aula, dando a oportunidade aos estudantes de manusear e analisar animais fixados, em um laboratório, por exemplo, e de terem aulas externas, seja em unidades de conservação ou em excursões a locais próximos, como hortas ou o espaço externo do colégio. Assim, o professor conseguiria estimular os alunos com uma abordagem mais ativa e personalizada, o que poderia compensar a falta de tempo dedicado à Zoologia no ensino básico (BESSA et al., 2018).

Uma outra abordagem didática na construção do conhecimento no ensino de zoologia é o uso de recursos lúdicos, como a utilização do jogo didático. Trindade et al. (2018), observaram que com o uso de jogos didático-pedagógicos no ensino, os alunos tiveram melhor aproveitamento nesta disciplina. Como a 
zoologia é uma área que requer visualização e imaginação dos alunos, observou-se que integrar o jogo na aprendizagem facilitou a compreensão do tema, além de trazer divertimento, conhecimento, prazer e satisfação ao aluno, e os ajudou a aprender a trabalhar em equipe.

Desse modo, esse trabalho tem como objetivo realizar análise de literaturas publicadas sobre as metodologias para o ensino de Zoologia na educação básica.

\section{DISCUSSÃO TEÓRICA}

A discussão é baseada em 10 trabalhos (Quadro 1) encontrados no site Google Acadêmico (Google scholar) entre o período de 2012 até 2021 . O critério utilizado para a seleção dos trabalhos aqui analisados levou em consideração a abordagem clara de metodologias para o ensino de zoologia. As palavras chaves utilizadas para a busca dos trabalhos foram as seguintes: ensino; material didático; metodologia; zoologia.

Quadro 1: Artigos utilizados para análise das metodologias pedagógicas utilizadas no ensino de zoologia.

\begin{tabular}{|l|l|l|}
\hline \multicolumn{1}{|c|}{$\begin{array}{c}\text { Ano de } \\
\text { publicação }\end{array}$} & \multicolumn{1}{|c|}{ Título do trabalho } & \multicolumn{1}{c|}{ Autores } \\
\hline 2012 & $\begin{array}{l}\text { Modelagem didática tridimensional de artrópodes, como método para ensino de ciências e } \\
\text { biologia. }\end{array}$ & BRITO \\
\hline 2013 & Metodologias e estratégias utilizadas para o ensino de zoologia. & $\begin{array}{l}\text { BASTOS } \\
\text { JÚNIOR }\end{array}$ \\
\hline 2014 & O uso de fotografias como estratégia para o ensino de zoologia. & PEREIRA \\
\hline 2015 & Ensino de zoologia: concepções e metodologias na prática docente. & RICHTER \\
\hline 2016 & $\begin{array}{l}\text { O potencial didático do folclore como ferramenta no ensino de zoologia na educação } \\
\text { básica: uma proposta estimuladora. }\end{array}$ & $\begin{array}{l}\text { MAGALHÃES et } \\
\text { al. }\end{array}$ \\
\hline 2017 & Ensino de zoologia em foco: interações e atividades investigativas. & DIAS et al. \\
\hline 2018 & Modelos anatômicos tridimensionais como metodologia de ensino em zoologia. & $\begin{array}{l}\text { SGANZELLA et } \\
\text { al. }\end{array}$ \\
\hline 2019 & $\begin{array}{l}\text { Inovações didáticas no ensino de zoologia: enfoques sobre a elaboração e comunicação de } \\
\text { relatos de experiências como atividades de aprendizagem. }\end{array}$ & ALMEIDA et al. \\
\hline 2020 & $\begin{array}{l}\text { Jogos didáticos para o ensino de zoologia no ensino médio: relato de experiência no } \\
\text { município de Ingá-PB. }\end{array}$ & SANTOS et al. \\
\hline 2021 & $\begin{array}{l}\text { Planejamento e construção de mapas conceituais em Zoologia: evidenciando a descrição } \\
\text { taxonômica e a divulgação sobre biodiversidade. }\end{array}$ & ALMEIDA et al. \\
\hline
\end{tabular}

O trabalho desenvolvido por Brito (2012) sugere a produção de modelos tridimensionais com massa de biscuit que simulam a estrutura corporal dos artrópodes como recurso didático para ensino de zoologia. Sobre a utilização deste material para a facilitação do ensino de zoologia, o autor afirma que,

A utilização de modelos didáticos tridimensionais é uma alternativa que deve ser estimulada nos estabelecimentos de ensino, pois promove a relação do conteúdo estudado com aulas práticas, onde os alunos podem observar e aplicar os termos e conceitos conhecidos em sala de aula, tornando o conteúdo mais assimilável e compreensível. (BRITO, 2012)

A utilização de massa de biscuit, massa de modelo infantil e massa do tipo epóxi têm sido amplamente utilizada por diversos educadores para a produção de recursos didáticos que facilitem a percepção dos estudantes sobre as estruturas morfológicas dos insetos, por exemplo. Matos et al. (2009) afirma que esses materiais possibilitam que os estudantes participantes das atividades de produção deles sejam eficazes durante o processo de aprendizagem. Além disso, os estudantes não só adquirem conhecimento científico a respeito do tema como também desenvolvem sua criatividade e a capacidade de trabalhar em equipe. 
Em seu trabalho de conclusão de curso, Bastos Junior (2013) afirma que,

Recursos didáticos como, multimídias e computadores permitem a introdução de uma série de informações sobre animais que nunca ou raramente são apresentados nos livros didáticos. Assim sendo, estes recursos didáticos têm papel importante na aprendizagem do aluno. (BASTOS JUNIOR, 2013)

Não são todas as instituições de ensino, principalmente as da rede pública, que possuem recurso multimídia para utilização como recurso didático de ensino. Entretanto, os professores que atuam em instituições de ensino que possuem computadores disponíveis com acesso à internet para a utilização como recurso didático devem explorar esse meio pelo qual os estudantes podem ter contato com imagens de animais que não são apresentados em seus livros didáticos. De acordo com Eugênio (2012) esses recursos são capazes de ensinar de forma "diferente e sofisticada" permitindo que o estudante possa treinar sua habilidade com as tecnologias usuais ao mesmo tempo em que aprende conceitos importantes sobre os animais e consegue identificá-los de maneira mais fácil, quando comparado ao material didático.

Analisando o trabalho de Pereira (2014), nota-se que a autora utilizou de fotografia para facilitar o processo de ensino e aprendizagem em zoologia com a utilização de fotografia. A autora afirma que,

O uso de fotografias como uma estratégia de ensino de Zoologia no Ensino Fundamental mostrou-se um eficiente recurso para estimular uma melhor compreensão dos conteúdos referente ao universo dos animais porque permite a apresentação e exploração de ricos detalhes da morfofisiologia e bioma ao qual o animal pertence. O que também caracteriza a fotografia como esclarecedora, ao oferecer mais informações sobre os animais através de códigos visuais, é o fato de ela permitir acessar conteúdos que, num primeiro momento, sem ela, seria abstrato para os alunos, difíceis de serem demonstrados pelo método tradicional, baseado, puramente, na fala do professor dirigida ao aluno. (PEREIRA, 2014)

Sobre a utilização de fotografia como recurso didático, os autores Santos et al. (2018) também afirmam que é um método "eficiente e eficaz" principalmente se for bem administrada durante a atividade proposta, pois visualizar de forma clara determinados aspectos do ensino, neste caso os animais e onde vivem é muito mais atraente do que ficar apenas imaginando, principalmente porque os estudantes talvez nunca tenham visto todos os animais abordados durante o ensino de zoologia ou em seu material didático.

O trabalho elaborado por Richter (2015) visa questionar o ensino de zoologia na atualidade, o autor observa que,

O Ensino de Zoologia encontra-se em uma inércia didática, pedagógica e metodológica. Mudanças nesse processo são possíveis, mas sob uma perspectiva transformadora e consistente. Para que o Ensino de Zoologia adquira um status de prática transformadora são necessárias mudanças ideológicas, individuais, coletivas e políticas, formativas e curriculares [...]. Os docentes devem ser instigados a desenvolver a prática reflexiva e crítica, devem ser preparados para confrontar suas ideologias, e também investigar suas próprias práticas. (RICHTER, 2015)

A observação feita pelo autor é pertinente, pois os recursos didáticos podem ser maravilhosos, as metodologias de ensino propícias para o público, mas nada disso terá valor se o educador não tiver um planejamento realista sobre as necessidades de seus estudantes. Além disso, é necessário que o educador tenha conhecimento do tema abordado para que ele possa escolher o melhor recurso didático que facilitará seu processo de ensino. É por isso que Prata et al. (2019) sugerem que os educadores realizem melhoras em sua forma de ensinar sempre que for possível, para não cair na falsa ideia de que o conhecimento adquirido 
durante sua graduação é o suficiente. Um meio pelo qual os educadores podem buscar melhorias em sua qualidade de ensino e desenvolvimento da criatividade é por meio de cursos de formação continuada, como uma pós-graduação, capacitação e/ou cursos de extensão.

O trabalho desenvolvido por Magalhães et al. (2016) ressalta que os recursos didáticos utilizados no ensino de zoologia,

Não pode, e nem tem a pretensão de, substituir o livro didático no desenvolvimento dos temas em questão. Ela pode atuar apenas como mais um recurso para complementar a explicação, ou como um ponto de partida para debates, experimentos ou outras atividades que o(a) professor(a) queira desenvolver em sua abordagem do conteúdo. (MAGALHÃES et al., 2016)

As aulas práticas, os jogos didáticos, filmes, atividade lúdicas, recurso tecnológicos e diversos outros métodos pelos quais os educadores prometem ensinar devem ser utilizados como método adicional de ensino aos elementos que já são utilizados, como as aulas teóricas e o livro didático, mas nunca como substituição destes, visto que cada metodologia apresenta relevância no processo de ensino. Sobre a importância dos diferentes recursos didáticos para o ensino de zoologia, Pinheiro et al. (2020) apontam que uma coleção didática de animais taxidermizados conservados é possível que os estudantes se sintam sensibilizados para o cuidado e preservação de ambientes naturais e costeiros que vêm sofrendo um processo constante de degradação.

A pesquisa de Dias et al. (2017) nos apresenta a necessidade de potencializar o ensino da zoologia com a inserção de atividades investigativas, que auxiliem na compreensão dos processos científicos envolvidos na classificação dos animais. Os autores dizem que,

As interações realizadas durante a atividade investigativa possibilitam a construção da compreensão para a necessidade de classificação dos animais no sentido de facilitar o estudo da grande diversidade que existe e tais informações podem ser utilizadas em situações práticas, como tratamento de doenças, preservação adequada de espécies, identificação de animais peçonhentos, etc. (DIAS et al., 2017)

A proposta de inserir os estudantes em atividades que utilizem os métodos de pesquisa científica colabora para que esses desenvolvam seu senso crítico de investigação. De acordo com Santos et al. (2008), a metodologia científica permite que os estudantes possam tornar-se personagens ativos no processo de ensino, indo contra o que costumeiramente ocorre, pondo o estudante como personagem passivo diante do processo de ensino, sentado ouvindo o professor falar ou utilizar o quadro. Não que isso seja um problema, é claro. Mas os métodos de ensino não devem limitar-se a esses métodos.

A pesquisa de Sganzella et al. (2018), assim como Brito (2012), também propõe a utilização de modelos tridimensionais que mostram as divisões corporais de artrópodes utilizando como materiais papel, isopor e tinta guache. Sobre a utilização desse recurso, ou autores afirmam que,

Os relatos dos alunos evidenciaram que a atividade lúdica tornou a aula mais atrativa e promoveu a fixação do conteúdo, possibilitando diferenciar as classes e suas divisões corporais. Esse tipo de metodologia trabalha o pensamento científico, crítico e criativo elencados como competência da Base Nacional Comum Curricular a ser estimulada na educação básica. (SGANZELLA et al., 2018)

Fernandes et al. (2014) também afirmam que as atividades lúdicas podem facilitar o processo de ensino e aprendizagem, por isso é importante que os educadores façam uso destas constantemente, com o 
objetivo de proporcionar uma "formação integral do educando".

Uma análise no trabalho de Almeida et al. (2019) permitiu observar que essas atividades realizadas com os estudantes e a utilização dos materiais didáticos devem ser documentadas para que outros professores possam reproduzir e melhorar seu processo de ensino. Sobre esses registros, os autores sugerem que,

As fontes de documentação tornam-se úteis para motivar os estudantes, não somente no processo de apreensão dos conteúdos específicos, mas para enriquecerem seus currículos durante o período de formação na universidade, demonstrando, com isso, a importância dos conhecimentos vivenciados frente a outras realidades acadêmicas. (ALMEIDA et al., 2019)

Esse achado nos faz refletir sobre a importância do registro de nossas pesquisas e sobre a necessidade de publicá-las para que alcancem o maior número de pessoas interessadas possíveis, como sugere Nornberg et al. (2014). Outros educadores que não possuem tanta criatividade para desenvolver diferentes tipos de materiais didáticos podem ser inspirados por outros trabalhos para desenvolver seus próprios materiais ou reproduzir algo já existente, a fim de tornar suas aulas mais interessantes e facilitadas por esses materiais.

O trabalho de Santos et al. (2020) apresentou como método lúdico para o ensino de zoologia a utilização de jogos didáticos. Sobre essa proposta os autores observaram que,

A construção e aplicação de jogos didáticos nas aulas de biologia é uma alternativa viável para o processo de ensino e aprendizagem, principalmente relacionados à temática de Zoologia. Os resultados demonstraram que os conhecimentos adquiridos sobre os táxons foram mais efetivos após a aplicação dos jogos didáticos, que favoreceram a participação e o interesse dos educandos pela aula. (SANTOS et al., 2020)

A utilização de jogos como material de ensino na área de biologia vem sendo explorada por diversos autores com sucesso. Campos et al. (2003) são pesquisadores que chegaram à mesma conclusão afirmando que além de favorecer o processo de aprendizagem fazendo com que o estudante aprenda e absorva determinado conhecimento, a utilização dos jogos torna essas atividades mais prazerosas, motivando os estudantes na interação com o conteúdo, com as atividades, no desenvolvimento de seu raciocínio e de suas capacidades de argumentação.

O último trabalho analisado foi desenvolvido por Almeida et al. (2021), já mencionado anteriormente em outro trabalho. Nesta proposta, os autores apontam a utilização de mapas conceituais como recurso didático para o ensino de zoologia, principalmente quando se aborda grupos animais pouco conhecidos, como os metazoários invertebrados, por exemplo. Eles afirmam que,

Ao inserir os mapas conceituais, para abordar sobre os conteúdos, enriquece-se a prática pedagógica e conecta-se ao estudo em processo, instrumentos concretos, visualizados como fontes de inspiração e exemplos a serem seguidos, promovendo situações para que outras produções sejam desenvolvidas a cada momento em que a experiência é proposta. (ALMEIDA et al., 2021)

Diversos autores apontam que os mapas conceituais são importantes para o processo de ensino de aprendizagem visto que relacionam determinado conceito com a estrutura do conhecimento o qual esse conceito pertence, de forma que facilita a compreensão e absorção dos conceitos a curto prazo, permitindo 
que os educadores possam prosseguir com as atividades sem delongar muito nos conceitos teóricos (ROSA et al., 2015; SILVEIRA, 2004; NOVAK, 2000).

\section{CONCLUSÕES}

Por meio desta análise bibliográfica reflexiva, é possível concluir que a utilização de fotografias, estruturas tridimensionais feitas com massa de modelar, jogos didáticos, computadores ou aparelho de celular com acesso à internet e mapas conceituais são recursos didáticos que colabora com o processo de aprendizagem no ensino de zoologia e para o senso de investigação crítica das estudantes. O livro didático também é um importante material de ensino e deve ser explorado junto com outras metodologias, de acordo com cada proposta. Além disso, as atividades elaboradas devem ser documentadas para que outros educadores possam reproduzi-las, caso desejem.

Outro ponto a ser destacado é a necessidade de os educadores buscarem atualização profissional visando oferecer um ensino com mais qualidade, evitando a estagnação do processo educacional visto que cada geração apresenta necessidades e valores diferentes da geração anterior.

\section{REFERÊNCIAS}

ALMEIDA, E. A.; SANTOS, R. L.. Planejamento e construção de mapas conceituais em Zoologia: evidenciando a descrição taxonômica e a divulgação sobre biodiversidade. Brazilian Journal of Development, v.7, n.2, p.15500-15519, 2021.

ALMEIDA, E. A.; SANTOS, R. L.; MELO, G. S. M. C. D. D.; D'OLIVEIRA, R. G.. Inovações didáticas no ensino de zoologia: enfoques sobre a elaboração e comunicação de relatos de experiências como atividades de aprendizagem. Brazilian Journal of Development, v.5, n.6, p.6699-6718, 2019.

ARAÚJO, J. N.. Zoológico do CIGS: um espaço não-formal para a promoção do ensino de zoologia no contexto da Amazônia. In: SIMPÓSIO INTERNACIONAL DE EDUCAÇÃO EM CIÊNCIAS NA AMAZÔNIA - SECAM, 1. Anais. Universidade do Estado do Amazonas, Manaus, 2011.

ARAÚJO, O. L.. Uma abordagem diferenciada da aprendizagem de Sistemática filogenética e taxonomia zoológica no Ensino Médio. In: CONGRESSO NACIONAL DE EDUCAÇÃO: I SEMINÁRIO INTERNACIONAL DE REPRESENTAÇÕES SOCIAIS, SUBJETIVIDADE E EDUCAÇÃO, 10. Anais. 2011.

BASTOS JÚNIOR, P. S.. Metodologias e estratégias utilizadas para o ensino de zoologia. Monografia (Bacharelado) Universidade de Brasília, Brasília, 2013.

BESSA, E.; ALVES, A.; OLIVEIRA, A.; NERY, B.; SOUZA, C.; ALVES, F.; FERREIRA, I.; RUAS, J.; BARBOSA, J.; TAVARES, K.; CRISTINA, K.; GALENO, L.; DUARTE, M.; SILVA, P.; NUNES, S.; SILVA, V. M.; MOREIRA, W. S.; RESENDE, P. V.. The Teaching of Zoology at the New Common National Base Curriculum. Boletim Informativo-Sociedade Brasileira de Zoologia, v.40, n.124, p.9-12, 2018.

BRITO, C. H.. Modelagem didática tridimensional de artrópodes, como método para ensino de ciências e biologia.
Revista Brasileira de Ensino de Ciência e Tecnologia, v.5, n.3, 2012.

CAMPOS, L. M. L.; SERAFIM, N. T.; TEIXEIRA, M. R.; FALONE, S. Z.. A produção de jogos didáticos para o ensino de ciências e biologia: uma proposta para favorecer a aprendizagem. Caderno dos núcleos de Ensino, v.47, p.47-60, 2003.

DIAS, M. G.; SESSA, P.. Ensino de zoologia em foco: interações e atividades investigativas. Enseñanza de Las Ciencias, p.5493-5498, 2017.

EUGÊNIO, T. J. B.. Utilização de uma ferramenta multimídia para identificação de artrópodes: avaliação de estudantes do ensino fundamental. Ciência \& Educação, Bauru, v.18, n.3, p.543-557, 2012.

FERNANDES, S. M. A.; MAVIGNIER, R. D.; SILVA, R. D. S.; SILVA, F. D. R.; DANTAS, S. M. M. M.. Baralho didático: temas de biologia para ensino médio. Revista da SBEnBIO, n.7, 2014.

MAGALHÃES, O. M.; SALGADO, L. G. V.. O potencial didático do folclore como ferramenta no ensino de zoologia na educação básica: uma proposta estimuladora. Revista Urutágua, n.34, p.12-27, 2016.

MATOS, C. H. C.; OLIVEIRA, C. R. F.; SANTOS, M. P. F.; FERRAZ, C. S.. Utilização de modelos didáticos no ensino de entomologia. Revista de Biologia e Ciências da Terra, v.9, n.1, p.19-23, 2009

NORNBERG, M.; SILVA, G. F.. Processos de escrita e autoria sobre a ação docente enquanto prática formativa. Educar em Revista, n.54, p.185-202, 2014.

NOVAK, J. D.. Apreender a criar e utilizar o conhecimento: mapas conceptuais como ferramenta de facilitação nas 
escolas e empresas. Lisboa: Paralelo, 2000.

PEREIRA, R. S.. O uso de fotografias como estratégia para o ensino de zoologia. Monografia (Bacharelado) -

Universidade de Brasília, Brasília, 2014

PINHEIRO, M. S.; SCOPEL, J. M.; BORDIN, J.. A importância de uma coleção didática de Zoologia para a sensibilização ambiental dos ecossistemas costeiros. Scientia cum Industria, v.8, n.1, p.7-11, 2020.

PRATA, E. G.; ARAÚJO, J. F.. As estratégias e metodologias no ensino de zoologia na zona rural do município de Breves PA. Biodiversidade, v.18, n.1, 2019.

RICHTER, E.. Ensino de zoologia: concepções e metodologias na prática docente. Monografia (Bacharelado) Universidade Federal da Fronteira Sul, Chapecó, 2015.

ROSA, I. S. C.; LANDIM, M. F.. Mapas conceituais no ensino de Biologia: Um estudo sobre aprendizagem significativa. Scientia Plena, v.11, n.3, 2015.

SANTOS, C. M. D.; CALOR, A. R.. Ensino de biologia evolutiva utilizando a estrutura conceitual da sistemática filogenéticaI. Ciência \& Ensino, v.1, n.2, 2008.
SANTOS, I.; FARIAS, F. L. B.; MELO, L. D. M.; SILVA, A. C. B.; FREITAS, D. L.; ELEUTÉRIO, D. A.; BARROS, A. T.. Jogos didáticos para o ensino de zoologia no ensino médio: relato de experiência no município de Ingá-PB. Brazilian Journal of Development, v.6, n.5, p.27076-27086, 2020.

SANTOS, K. M.; MIRANDA, J. C.; GONZAGA, G. R.. A fotografia como recurso didático. Educação pública, Rio de Janeiro, v.18, 2018.

SANTOS, S.; TERÁN, A.. Condições de ensino em zoologia no nível fundamental: o caso das escolas municipais de Manaus-AM. Revista Areté: Revista Amazônica de Ensino de Ciências, v.6, n.10, p.01-18, 2017.

SGANZELLA, B. E.; TAKIZAWA, R. H.; SOUZA, A. E. M. Modelos anatômicos tridimensionais como metodologia de ensino em zoologia. Anais do Fórum de Iniciação Científica do UNIFUNEC, v.9, n.9, 2018.

SILVEIRA, F. P. R. A.. A aprendizagem significativa na formação de professores de biologia: o uso de mapas conceituais. Revista Brasileira de Pesquisa em Educação em Ciências, v.4, n.3, 2004.

A CBPC - Companhia Brasileira de Produção Científica (CNPJ: 11.221.422/0001-03) detém os direitos materiais desta publicação. Os direitos referem-se à publicação do trabalho em qualquer parte do mundo, incluindo os direitos às renovações, expansões e disseminações da contribuição, bem como outros direitos subsidiários. Todos os trabalhos publicados eletronicamente poderão posteriormente ser publicados em coletâneas impressas sob coordenação da Sustenere Publishing, da Companhia Brasileira de Produção Científica e seus parceiros autorizados. Os (as) autores (as) preservam os direitos autorais, mas não têm permissão para a publicação da contribuição em outro meio, impresso ou digital, em português ou em tradução. 